\title{
A High-Resolution Resistive Probe for Nonlinear Analysis of Two-Phase Flows
}

\author{
L. Cantelli, A. Fichera, and A. Pagano \\ DIIM, Università Degli Studi di Catania, Viale A. Doria n. 6, 95125 Catania, Italy \\ Correspondence should be addressed to A. Fichera, afichera@diim.unict.it
}

Received 11 April 2011; Accepted 27 June 2011

Academic Editor: D. W. Pepper

Copyright ( $\odot 2011$ L. Cantelli et al. This is an open access article distributed under the Creative Commons Attribution License, which permits unrestricted use, distribution, and reproduction in any medium, provided the original work is properly cited.

Two-phase flow dynamics are highly complex, due to the strong coupling of various independent mechanisms and as demonstrated by the existence of a variety of flow patterns. The adoption of appropriate tools for nonlinear time series analysis tools may lead to a deeper insight in this complexity but requires high quality time series. This study describes a procedure appositely assessed in order to realise an impedance void fraction sensor of resistive type characterised by high-spatial and -temporal resolution. These characteristics have been accomplished through an appropriate geometrical design of the probe electrodes, aiming at obtaining a thin measurement volume so to improve the probe spatial resolution, and through the electronic assessment of the data acquisition system, improving its temporal resolution. A new calibration procedure has been also defined, based on an estimation of void fraction through a code for automatic extraction of bubble contours and the correction of image distortions.

\section{Introduction}

Gas-liquid two-phase flows in pipes are at the basis of a wide variety of heat and mass transfer applications, ranging from power generation to chemical, processing, and oil plants. This kind of flows is characterised by high complexity, as a consequence of the strong coupling of several mechanisms and of the dependence on various factors, the most important of which are the differential action of gravity on the two-phases and the effect of shear and surface tension forces at their interface. In particular, depending on the hydrodynamic equilibrium at the interface between the two phases, several different flow patterns can be identified, each of which can be characterised in terms of the peculiar distribution of the two phases and of pressure drop. The type of flow pattern represents one of the fundamental factors governing the dynamics of two phase systems; therefore, flow patterns identification and classification are of primary importance for both engineering design purposes and for the monitoring of applications involving two-phase flows.

Though several different names and classifications have been proposed by researchers, some flow patterns have been recognized as typical and reported in several classifications, such as works in [1-5]. For the case of vertical pipes consid- ered in the present study, the basic flow pattern classification distinguishes between bubbly, slug, churn, and annular flow.

In the bubbly flow small diameter gas bubbles are dispersed in the liquid phase. The main characteristic is that coalescence phenomena, though present, are unable to produce gas bubbles occupying the pipe section, as it happens in the slug flow.

The slug flow consists in an intermittent flow of Taylor bubbles alternated to liquid slugs. Moreover, the liquid slugs can be aerated or not, as a consequence of the entrainment of small gas bubbles. The respective flow rates of the two phases are determinant for the development of the bubble, which may range from very short to elongated, depending on the relative importance of the three main parts in which the bubble can be subdivided. These are

(i) the head region, where the fraction of the pipe section occupied by gas rapidly grows from zero to approximately the whole section, that is, except for the thin liquid film separating the gas from the tube wall;

(ii) the central region, where the pipe section is mainly occupied by the gas phase, again except for the liquid film at the wall, which is often subjected to 
longitudinal oscillations, especially if the central region is sufficiently developed;

(iii) the tail region, where the gas occupying the pipe section abruptly falls to approximately zero, that is, except for the shedding of relatively small bubbles in the liquid slug that follows the Taylor bubble.

Depending on the development of the central region of the bubble, it is possible to draw a main distinction in the class of slug flow. In particular, cap flow occurs when the head and tail regions of the bubble are consecutive and no central region can be distinguished. Cap flows usually occur at relatively low values of the gas mass flow rate; therefore, the cap-shaped bubbles are usually separated by long liquid slugs. In the proper slug flow, the axial development of the head, central, and tail regions of the bubble is comparable, whereas the plug flow is characterised by elongated Taylor bubbles, that is, it occurs when the extension of the central region is markedly predominant with respect to the head and tail regions. The distinction between slug and plug flow remains somewhat controversial; nonetheless, plug flow occurs at relatively higher values of the gas mass flow rate and, therefore, the liquid slugs separating the gas plugs are usually shorter and more aerated than those separating the gas bubbles in slug flow.

The churn flow is a highly turbulent flow that occurs for higher values of the gas mass flow rate. In this flow pattern, the waving liquid film still drains down the wall, as in elongated Taylor bubbles, but occasionally bridges the tube wall forming short and highly aerated slugs. Finally, the annular flow consists of a thin annular film of liquid on the tube wall on which small ripples, occasionally interspersed with large disturbance waves, flow in a regular manner along the tube wall. These flow patterns occur when the gas mass flow rate is high enough to guarantee the stability of the liquid-gas interface.

The characterization of the various flow patterns can be performed on the basis of the analysis of void fraction-related signal fluctuations. In fact, under normal gravity conditions, the void fraction represents the most important variable for the description of the distribution of the two-phases within the pipe section, and is therefore a fundamental parameter for the identification and classification of the flow regime. The accuracy of the classification strategy strongly depends on the spatial and temporal resolution of the technique adopted for the measure of the void fraction. Several of these techniques have been proposed; the most common are based on the measure of the electrical impedance of two phase mixtures $[2,6,7]$, of the optical scattering of the interface between the two phases [8], or of the pressure difference observed along a specified piece of the pipe [9].

The design of optical sensors allow to perform local measurement but is severely affected by disturbances caused by the nonlinearity of the scattering on curved interfaces; therefore, in general, the quality of their experimental time series is very poor. In contrast, differential pressure sensors are not sensitive to phenomena induced by the curvature of the interface between the two phases but cannot be designed in order to obtain a reliable local measurement, as they require the two pressure tips to be placed at a sufficient distance along the pipe axis. Moreover, the structure of the time series of neither optical nor differential pressure sensors can be directly related to the physical distribution of the two phase within the flow pattern.

In contrast to the previous studies, impedance measurements seem to be particularly reliable [8]. They are nonintrusive and, most important, less dependent both on internal disturbances and external factors. Two main classes of impedance sensors have been proposed in the literature: resistive sensors $[2,6,10,11]$ and capacitance sensors $[7$, 9]. This kind of sensors presents some relevant advantages with respect to the previous. In particular, a strong correspondence exists between the shape of the oscillations of the experimental time series and the physical distribution of the two phase within the pipe section for the various flow patterns; this is remarkably verified for flow patterns characterised by pulsations of a relatively stable nature, such as, for example, for cap, slug, and plug flows, but also for annular flows with a wavy liquid film at the pipe wall. On the basis of this correspondence, for example, the identification of the passage of a bubble through the measurement volume, as well as its shape and length, is clearly reported in the time series. A second important advantage, partly depending on the previous, is the minor influence of secondary noisy phenomena, such as the dynamics of small gas bubbles dispersed in the liquid phase, which instead sensibly reduce the quality of the time series of both optical and differential pressure sensors.

In addition to the intrinsic complexity of two-phase flows, it is difficult to compare results of reported experiments due to limited information on measurement techniques as well as on calibration and validation procedures. In particular, the QCVs calibration procedure $[2,7]$ does not appear appropriate for instantaneous local void fraction measurements (i.e., measurements detected in a portion of the pipe sufficiently short to be reliably approximated to a section). In fact, this technique offers an exact measurement of the void fraction by trapping a portion of the flow in a clear tube. The relative amounts of air and water in the tube can then be directly measured to obtain a void fraction value to be compared to the mean value of the void fraction time series measured from the sensor over a time interval corresponding to the valves distance. If the closing time of the valves is less than $0.01 \mathrm{~s}$ the experimental error can be neglected [7]. In addition, the portion of the pipe between the two valves must be long enough to neglect the internal volume of the two valves. On the other hand, the QCVs approach does not allow the estimation of a local value of the void fraction; in other words, this value cannot be directly correlated with the time series measured by the sensor, but only with its mean value evaluated in a time window specified as the ratio between the axial length of the calibration section (i.e., the distance between either the QCVs or the pressure tips) and the flow pattern characteristic velocity.

The aim of the present study is to present a probe for void fraction measurement and the procedure used for its calibration. In particular, an impedance sensor of the 
resistive type has been specifically designed and realized with the aim of achieving high-spatial and temporal resolution. Such resolution is considered fundamental in view of the future adoption of non-linear time series analysis techniques for advanced characterization of the dynamics of two-phase flows. The proposed calibration procedure is based on the estimation of the void fraction directly from high resolution photos. The following section is devoted to the description of the experimental setup and is followed by the description of the characteristics of the sensor, the calibration procedure and the experimental campaign, reproducing the main flow patterns families and allowing to test the performance of the system.

\section{Experimental Apparatus}

Figure 1 represents a scheme of the experimental apparatus set up for the present study.

The liquid is supplied by means of a pump connected to a reservoir. The liquid flow rate can be varied up to $150 \mathrm{~L} / \mathrm{min}$ by means of a series of valves and bypasses placed at the outlet of the pump. An electromagnetic flow meter is used in order to measure the velocity and the mass flow rate of the water. The liquid is distilled water with sodium chloride $(1.5 \mathrm{~g} / \mathrm{L})$ in order to assure satisfactory conductivity. The temperature of the water in the reservoir has been maintained at $22^{\circ} \mathrm{C}$ $\left( \pm 0.2^{\circ} \mathrm{C}\right)$ in order to avoid the influence of temperature variation on conductivity measurement.

The air line is constituted from a pressure regulator $(0-$ 8 bar) and three air flow meters that can regulate the air flow rate in the range $0-200 \mathrm{~L} / \mathrm{min}$. The air is supplied to the mixing chamber by a pressurised tank fed by a compressor.

In order to allow the inspection of the flow pattern, the test section is constituted by a transparent vertical pipe of length $3 \mathrm{~m}$ and diameter $0.24 \mathrm{~m}$. At its basis there is a mixing chamber that connects the liquid and air lines. In order to allow the degassing of the working fluid an open tank is placed on top of the vertical pipe.

Two void fraction probes are placed at a distance of $5 \mathrm{~cm}$ from each other; the lowest of them is at $2.40 \mathrm{~m}$ from the mixing section, that is, at a distance greater than the entrance region (assumed 80 times the pipe diameter [12]) in order to assure a well-established flow regime at the measurement section. The two probes permit to estimate the velocity of bubble during the calibration procedure. A reference probe is placed upstream the mixing chamber on the liquid line. All the probes are connected to an electronic circuit and an appropriate data acquisition system.

2.1. Design and Calibration of a Void Fraction Resistive Probe. If a significant difference exists in the electrical properties of the two phases, the volume fraction of one of the phases in a two-phase mixture can be determined by measuring the mixture impedance. The impedance is made up of both resistance and capacitance; therefore, a main point in the design of an impedance void fraction sensor is the choice of the excitation frequency, which determines the dominance of the resistive or capacitive behaviour.

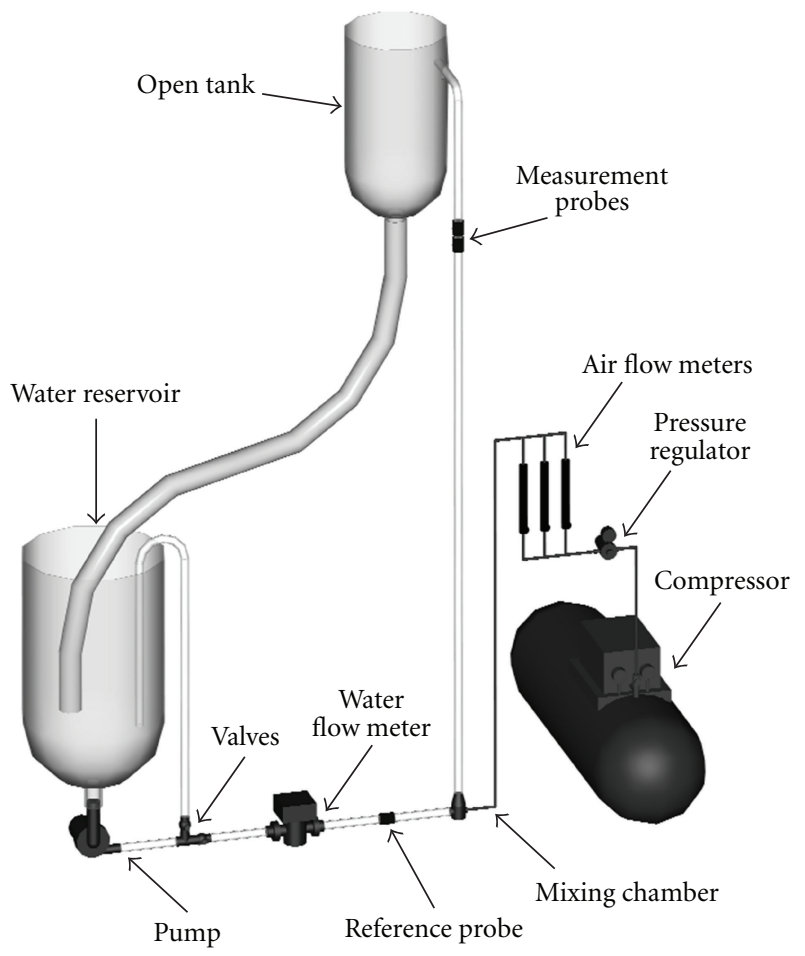

FIgURE 1: Experimental apparatus.

A mathematical model of an impedance probe can be briefly described as follows [13]. The impedance of a flow medium, $Z$, can be measured by two electrodes and can be expressed as

$$
Z(f)=\left[\frac{1}{R+\left(1 / i 2 \pi f C_{p}\right)}+i 2 \pi f C_{d}\right],
$$

where $R$ is the fluid resistance, $C_{p}$ is the capacitance due to the polarization of the fluid molecules at the electrodes, $C_{d}$ is the dielectric capacitance of the fluid, and $f$ is the excitation frequency at the electrodes. In the case of a twophase mixture, $R$ is a variable that depends on the difference of conductivity of the two phases (e.g., water and air for the present study). If the conductivity and the dielectric constant of the less-conductive phase (air) can be assumed negligible with respect to those of the conductive phase (water), $C_{p}$ depends on the dielectric constant of the conductive phases whereas $C_{d}$ is a function of the difference in the dielectric constants of the two phases, of the excitation frequency and of the void fraction. Therefore the void fraction can be determined by measuring either the resistance $R$ or the capacitance $C_{d}$ and, therefore, impedance sensors are usually classified as either conductive or capacitive sensors.

When the conductivity of the conductive phase is large, the measurement of capacitance $C_{d}$ requires the use of high excitation frequencies (over $1 \mathrm{MHz}$ ), in order to minimize the role of the resistance and eliminate the parasitic capacitance caused by polarization at the electrodes, as well as to reduce the effect of external disturbances on the measurement system. Nonetheless, in order to overcome 
complications in the electronics associated to high excitation frequencies, the measure of the resistance is preferred. In this case the influence of $C_{d}$ on measured impedance can be minimized by keeping the excitation frequency in a range depending on the electrical properties of the conductive phase. When water is considered as conductive phase such a range is $10-100 \mathrm{kHz}$. The choice of values higher than about $15 \mathrm{kHz}$ is preferred in order to eliminate the role of parasitic capacitance $C_{p}$.

The sensor that has been designed and realised for the present study is schematised in Figure 2. The probe design is similar to that proposed in $[2,14,15]$. It is made up by a pair of measuring electrodes that have been realised by means of gold-plated wires with a diameter of $0.6 \mathrm{~mm}$ forming two half rings facing one another, each spanning an $\operatorname{arc}$ of $90^{\circ}$. In addition, two pairs of gold-plated guard electrodes (identical to the previous) have been placed at a distance of $1 \mathrm{~mm}$ from the measuring pair on both its sides and maintained at the potential of the corresponding measuring electrodes. All the electrodes have been flush-mounted, in order to avoid disturbing effect on the flow.

The criterion of geometrical design has been chosen in order to minimise the measurement volume. In fact, the axial length of the measuring electrodes can be neglected with respect to the pipe diameter so that the measurement volume can be approximated to the pipe transversal section. This means that the proposed electrode design allows the observation of local two-phase phenomena on the pipe section, ensuring higher spatial resolution than that obtained by other geometries of analogous sensors [16, 17]. It is worth observing that the sensor does not produce accurate measures of small-scale void fraction variations, in particular those characterised by a length scale below $0.6 \mathrm{~mm}$. Such phenomena are typically associated to either dispersed bubbles, occurring both in the bubbly flow and in regimes characterised by aeration of the liquid phase, or the highfrequency and low-amplitude components of the oscillations in wavy liquid films.

The probe has been operated in the resistive range. In fact, a carrier frequency of $20 \mathrm{kHz}$ has been supplied by an external sine wave oscillator to both a measurement and a reference probe. The latter is a sensor identical to the measurement one that has been placed along the water line, as shown in Figure 1, in order to allow the elimination of the drift in void signals caused by possible changes in electrical properties of the flow medium.

Figure 3 shows the circuit used for signal processing. The instrumentation amplifier ensures high-dynamic response and perfect decoupling of the electronic circuit from the measuring section. The gain can be regulated by varying the $R_{G}$ value. The amplified output is applied to the electronic rectifier. A cut-off frequency of $200 \mathrm{~Hz}$ has been adopted in order to remove the carrier frequency and to avoid aliasing with the sampling frequency. The final output is sent to a PC-based data acquisition system at the sampling rate of $1000 \mathrm{~Hz}$, to allow the recording of the main void fraction fluctuations expected in the experiments.

In order to relate the resistive probe measurements directly to the actual instantaneous local void fraction, the calibration of the resistive sensor has been performed by comparing the value of the two-phase mixture conductivity measured by the sensor with the local diameter of the bubble as estimated by pictures taken by means of a highresolution camera. In this way each point of the impedance time series describing the passage of a gas bubble has been correlated with the fraction of air occupying the pipe section. The estimation of the local bubble diameter requires the consideration of image distortion phenomena, deriving from the simultaneous presence of refraction and reflection at the curved pipe walls and of the liquid film around the bubble. Under the assumption of symmetric axial distribution of the interface between the two phases, which approximately holds for most of the flow patterns, distortion phenomena have been simultaneously estimated leading to the assessment of the correction function reported in Figure 4, which allows to evaluate the actual bubble local diameter.

After the determination of the correction function, it has been possible to calibrate the sensor response through the estimation of the instantaneous local bubble diameter from pictures taken by a high-resolution camera focused on the portion of the pipe immediately before the resistive sensor.

During the calibration procedure, the experimental apparatus has been operated setting up the air and water flow rates within the range of stability of the slug flow. After that the regime condition has been established in the system, the time series corresponding to the passage of a bubble through the measuring section of the resistive sensor has been recorded and compared with the synchronized picture of the bubble.

A code has been developed in order to process the pictures, performing the automatic extraction of the bubble contour and the correction of distortions. The code output is the spatial distribution of the void fraction along the bubble, which is related to the temporal distribution of the void fraction measured by the resistive probe by means of bubble velocity. The velocity value has been determined through the evaluation of the delay in the crosscorrelation function between the time series measured by two resistive probes placed at known distance $(5 \mathrm{~cm})$.

Figure 5 allows to compare the resampled time series of the resistive probe and the samples of the local void fraction as estimated from the measure of the bubble diameter. In this way each piece of the time series of the resistive probe, describing the passage of a bubble, has been correlated with the instantaneous value of the fraction of air flowing through the pipe section.

This procedure has been repeated for several samples and results have been summarized in Figure 6, by plotting the void fraction versus the conductance time series. Finally, the calibration curve reported in Figure 7 has been obtained as the seventh-order polynomial curve interpolating the estimated void fraction for several bubbles.

2.2. Experimental Tests. After the sensor characterization an experimental campaign has been performed. In particular, a series of tests has been carried out by varying the air and water mass flow rates, in order to identify the main types of flow patterns through the analysis of void fraction time 

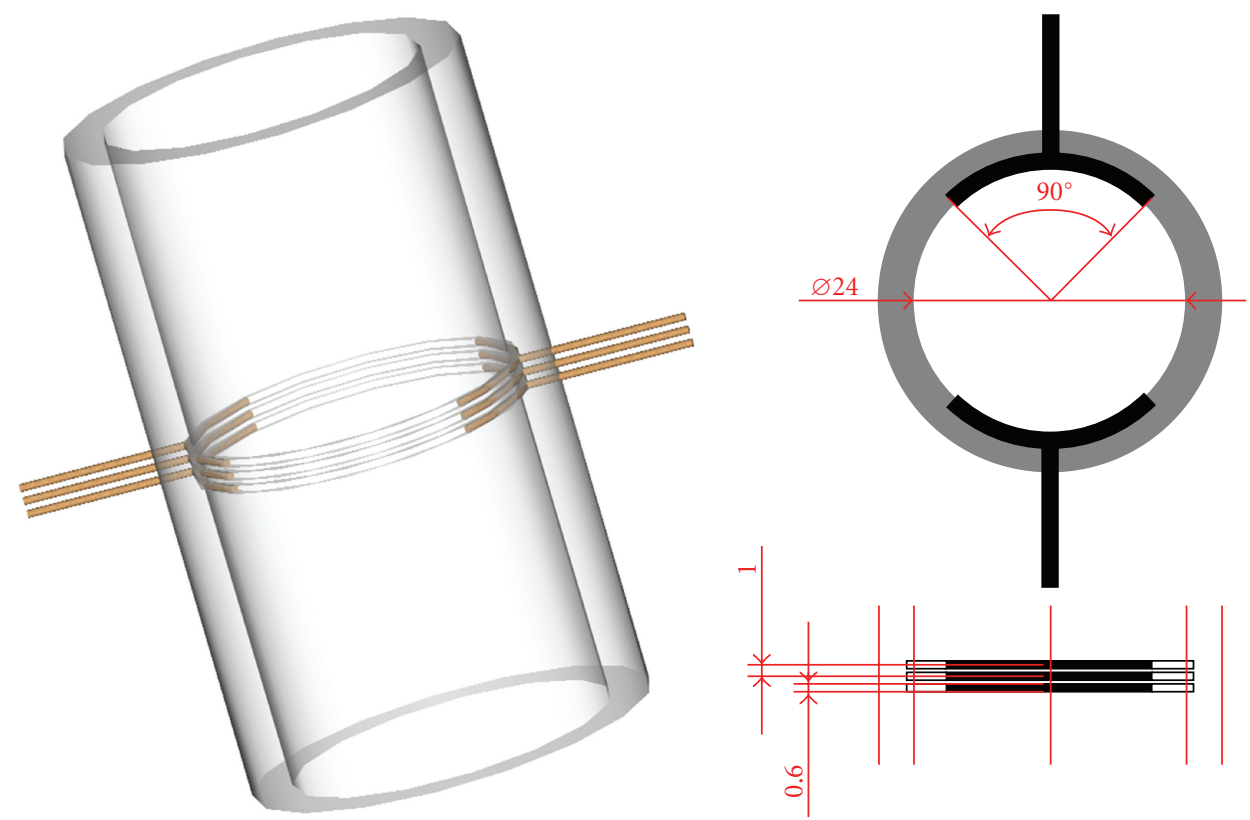

FIGURE 2: Schematic of adopted probes.

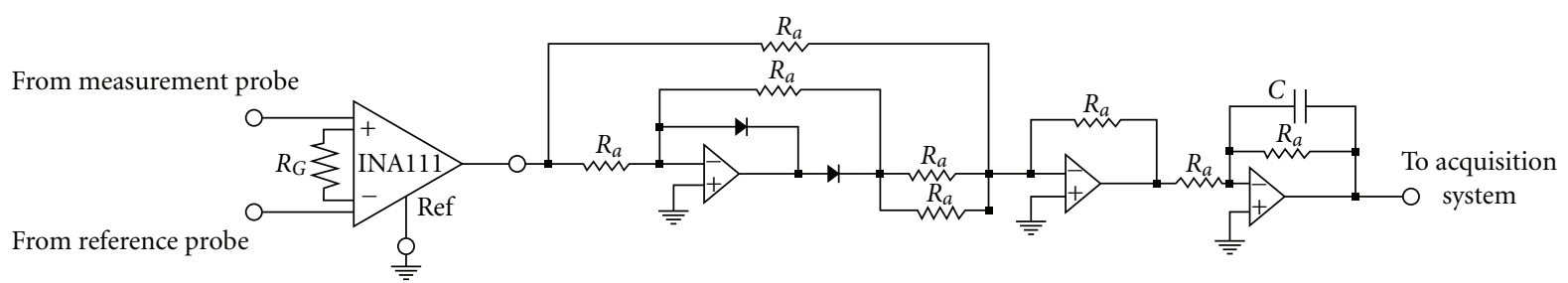

FIGURE 3: Electronic circuit for the probe.

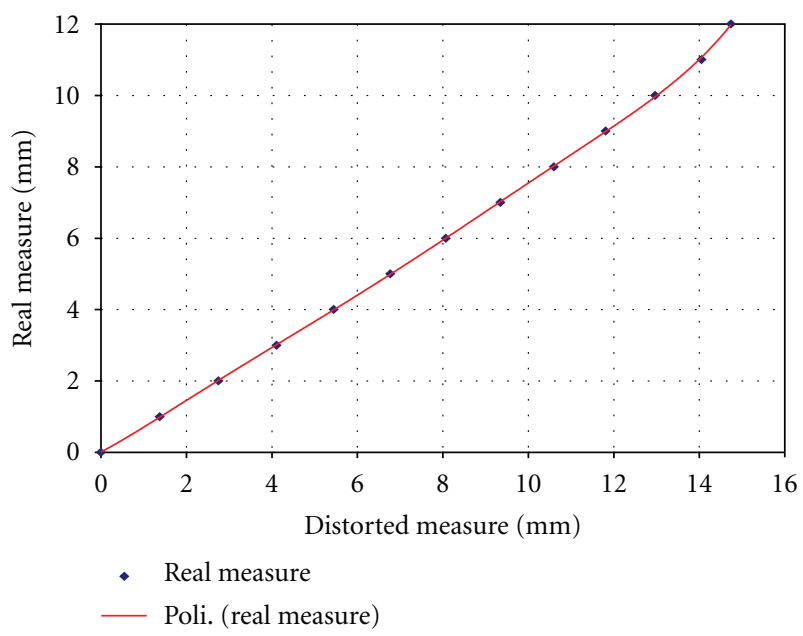

FIGURE 4: Correction function accounting for optical distortions.

series measured by the resistive probe. Table 1 shows the various operating conditions of the performed tests. Under these conditions different flow patterns have been observed to characterize the two-phase flow in the pipe.

In particular, for the sake of the result presentation, Table 2 reports the correspondence that has been established between typical flow patterns as reported in [2] and a restricted selection of testing conditions in Table 1.

\section{Void Fraction Time Series Analyses}

This section reports the results of the preliminary analyses of the experimental time series detected by the void fraction probe. The aim of these analyses is to verify the capability of the void fraction sensor to deal with the time evolution of the complex dynamical phenomena governing the distribution of the two phases under the various flow patterns. For synthesis, results will be discussed only for the experimental conditions reported in Table 2, chosen as representative of the main classes of flow patterns. Nonetheless, reported considerations have been found to be of general validity for the entire set of conditions detected during the experimental campaign.

At first, the experimental void fraction time series have been analysed in the time domain, as reported in Figure 8 . An important observation on the quality of these time series concerns the substantial lack of noise which, on the other hand, usually affects the experimental results reported in literature $[2,8,15,18]$. At the same time, the temporal evolution of the experimental time series appears sufficiently smooth, that is, the acquisition frequency of the void fraction 


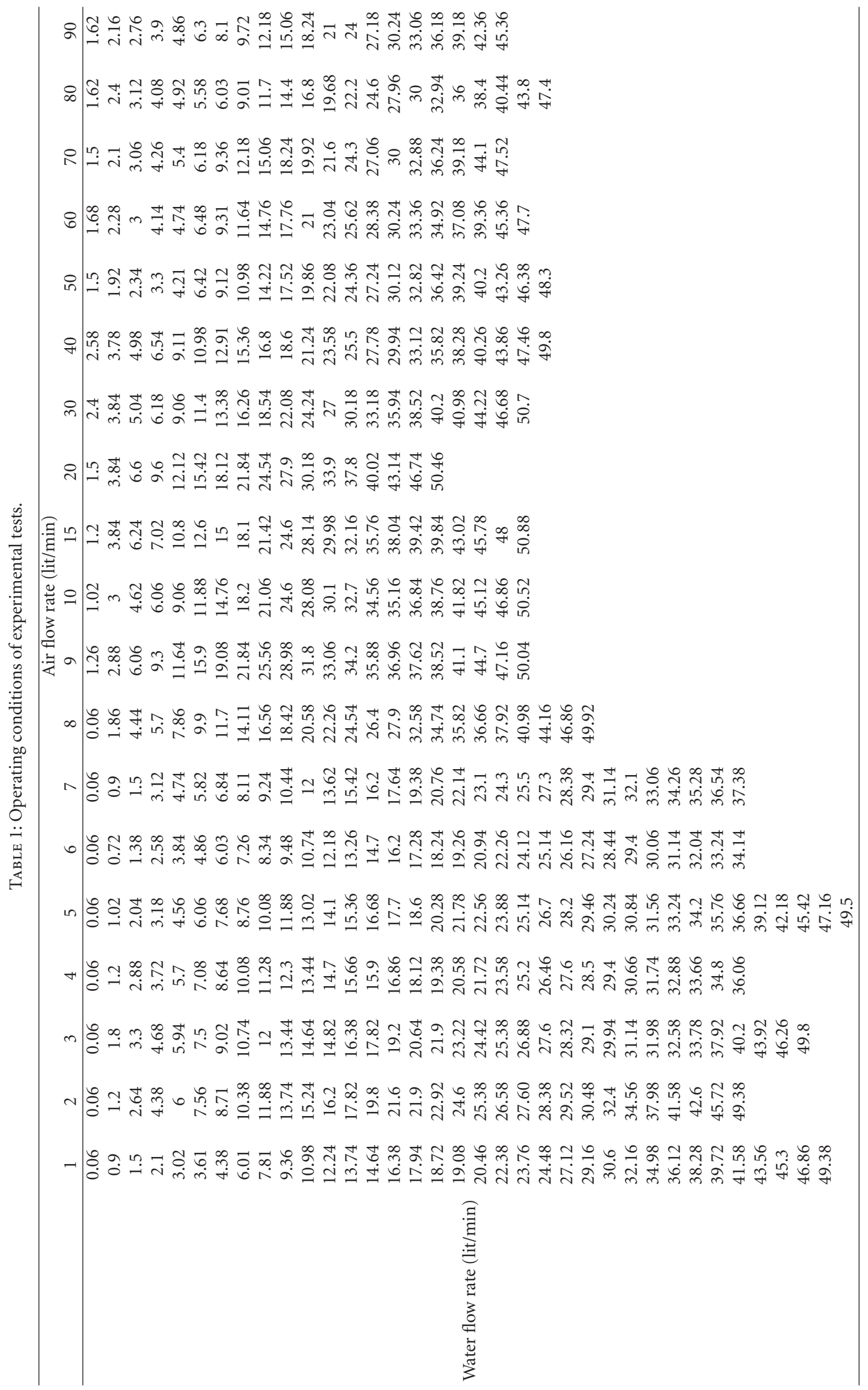




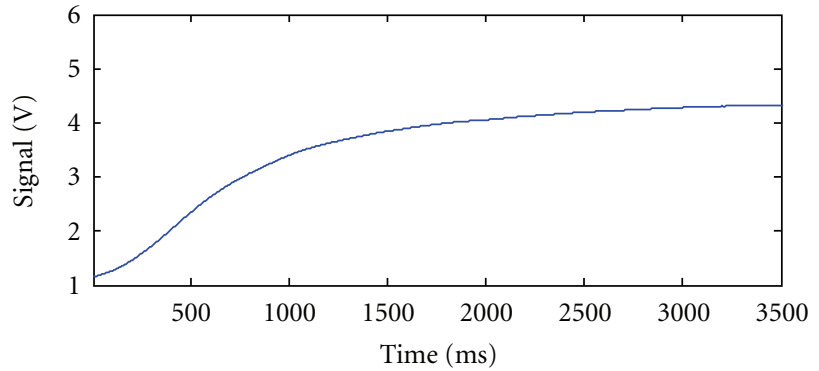

(a)

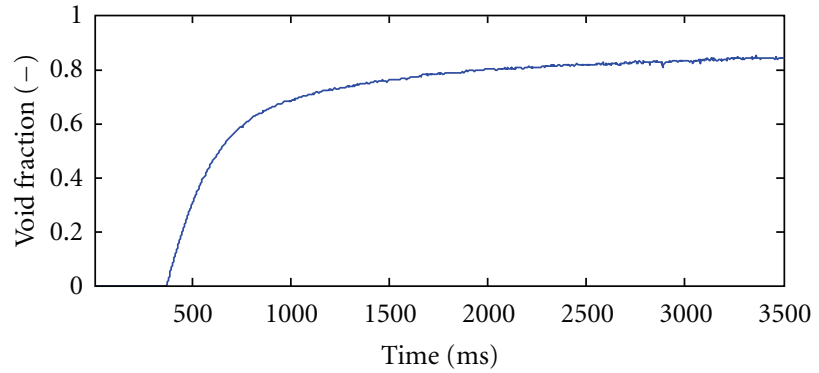

(b)

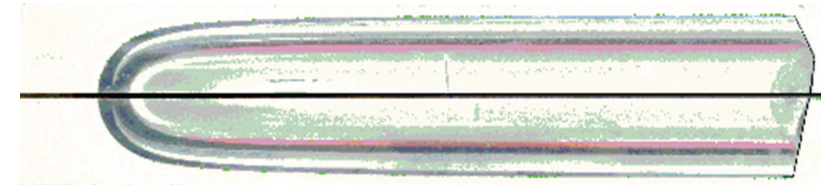

(c)

FIGURE 5: Comparison between the time series of the sensor (a) and the local void fraction estimation (b) obtained processing the picture of the bubble (c).

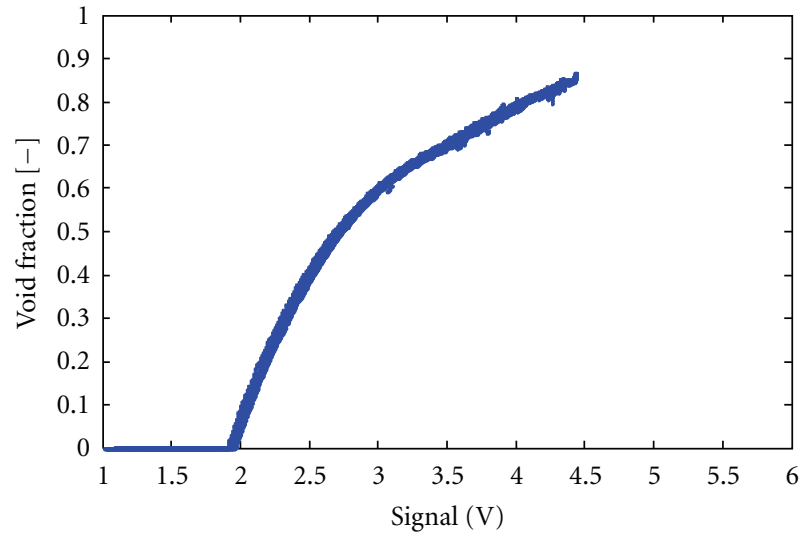

Figure 6: Calibration data for several bubbles detected under different operating conditions.

TABLE 2: Selection of testing conditions corresponding to typical flow patterns.

\begin{tabular}{lcc}
\hline Flow pattern & $\dot{m}_{\text {Water }}($ lit $/ \mathrm{min})$ & $\dot{m}_{\text {Air }}($ lit $/ \mathrm{min})$ \\
\hline Bubble & 30.60 & 1 \\
Cap & 10.98 & 1 \\
Slug & 2.40 & 1 \\
Plug & 2.40 & 10 \\
Churn & 3.06 & 40 \\
Annular & 1.62 & 90 \\
\hline
\end{tabular}

sensor is high enough to guarantee an appropriate description of the phenomena. In consideration of the thin measurement volume achieved with the architecture of the void fraction sensor, it is therefore possible to claim the general satisfactory performances of the sensor with respect to its spatial and temporal response. As a matter of fact, low noise influence and good spatial and temporal response represent

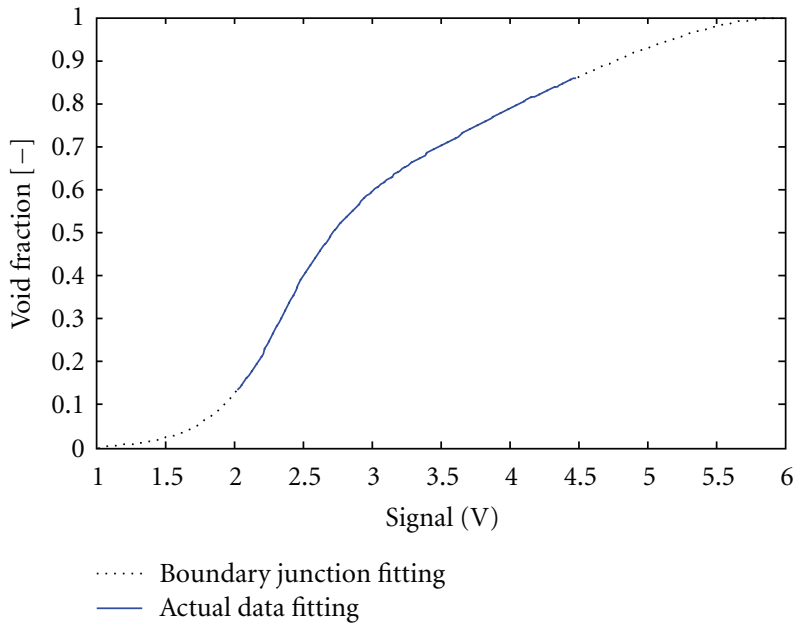

Figure 7: Calibration curve.

one of the main goals which the design and calibration of the void fraction sensor herein described wished to achieve.

Concerning the dynamical behaviour of the time series reported in Figure 8, it is worth observing that, though several important differences exist between the time series of most flow patterns, with the exception of bubble flow, all of the time series are characterised by relevant amplitude and frequency differences between consecutive oscillations, showing the non-periodical nature of the system dynamics. Nonetheless, the repetition of similar waveforms seems to be a common feature of the various flow patterns, indicating that some kind of recurrent dynamics can be distinguished for each flow pattern. This result agrees with the analyses of the autocorrelation and of the power spectral density distributions of the experimental time series reported in a previous preliminary study [19] for analogous flow patterns detected with the same experimental apparatus. In [19] it 


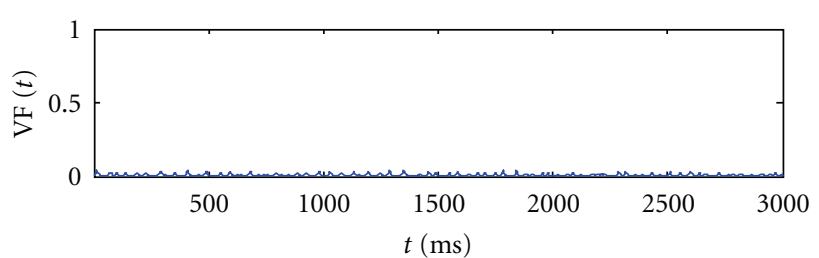

(a)

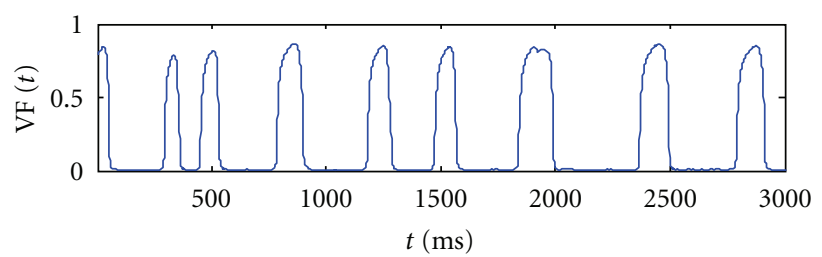

(c)

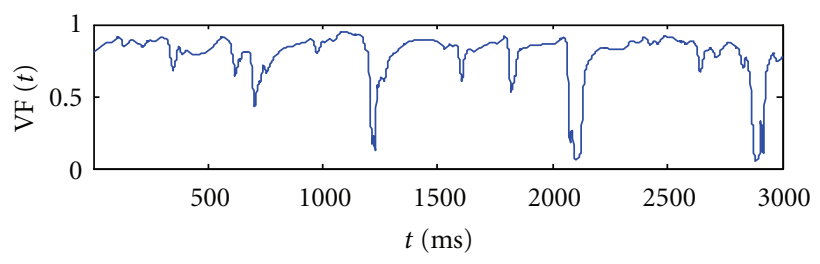

(e)

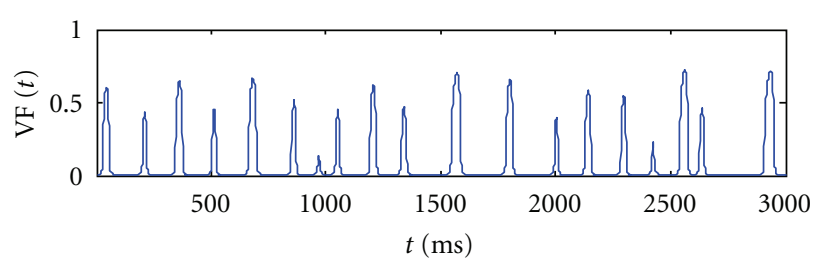

(b)

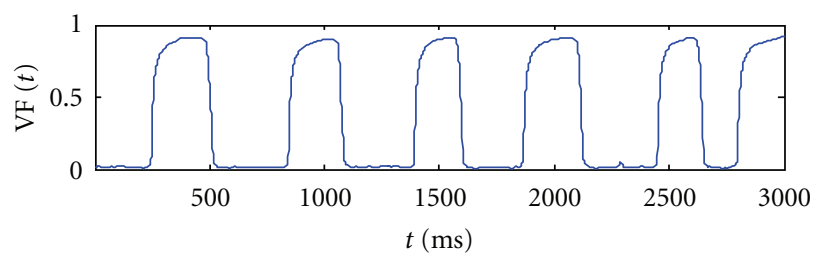

(d)

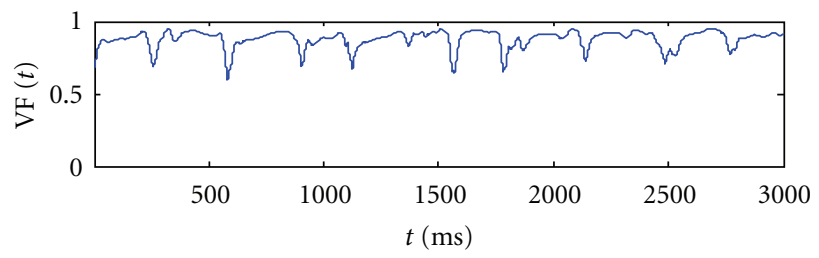

(f)

Figure 8: Experimental void fraction time series for the various flow patterns as reported in Table 2: (a) bubble flow; (b) cap flow; (c) slug flow; (d) plug flow; (e) churn flow; (f) annular flow.

was shown that, though a strong autocorrelation in the time series seems to indicate the deterministic nature of the experimental two-phase flow patterns, their complexity poses a limit to the validity of Fourier analysis and results in a typical broad-band power spectrum. As well known, both these observations hint at a deterministic source of chaotic dynamics, which cannot be satisfactorily described by means of previous linear tools.

Several studies [20-24] have claimed the existence of chaos in two phase flows but reported results are in general prone to relevant uncertainty due both to the general unsatisfactory spatial and temporal resolution of the experimental time series and to the strong influence of noise or of noiselike dynamics. Therefore, considering the satisfactory performance of the void fraction sensor described in this study in terms of both reduced noise influence and spatial and temporal time series resolution, [19] also reported the morphological analysis of the attractors of the experimental time series, evidencing the existence of a well-defined and regular structure in phase space, that is, a first important hint of deterministic chaotic behaviour.

In consideration of the innovations introduced both in the sensor design and construction as well as in the calibration of the sensor, part of the experimental analysis aims at validating the performance of the sensor through the comparison with similar sensors. In particular, in the present study, the focus has been posed on verifying the correspondence between the statistical distributions of the measurements detected by the sensor with those reported in literature for the same kinds of flow patterns. In fact, the analysis of the statistical distribution of the experimental time series is indeed among the preferred tool to classify and distinguish the various flow patterns.

Figure 8 reports the distributions of the Probability Density Function (PDF) for the time series of the void fraction measurements detected during the experimental test reported in Table 2 and plotted in Figure 8. From the analysis of Figure 9 emerges that the application of PDF analysis to the time series detected during the experimental campaign allows a clear classification of two-phase flow patterns. In fact, for each flow pattern, the PDF is characterised by a welldefined distribution. Moreover, what is particularly relevant for the aims of the present study is that the PDFs calculated from the experimental time series well-correspond to the distributions reported in literature for the same kind of flow patterns $[2,3,15]$. In other terms, the sensor performances are satisfactory not only in terms of spatial and temporal resolution and of low influence of noise, but also in terms of information content in view of flow pattern classification.

Therefore, future studies will be concerned on the exploitation of nonlinear tools both for the analysis of the experimental time series detected by means of the void fraction probe described in the present study and for the assessment of reliable criteria for flow pattern classification, based on an appropriate consideration of the complexity of the dynamics of two-phase flows. 


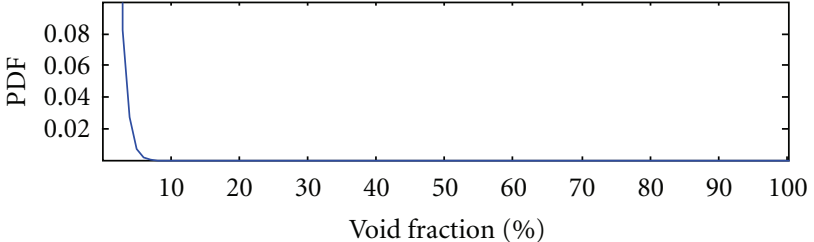

(a)

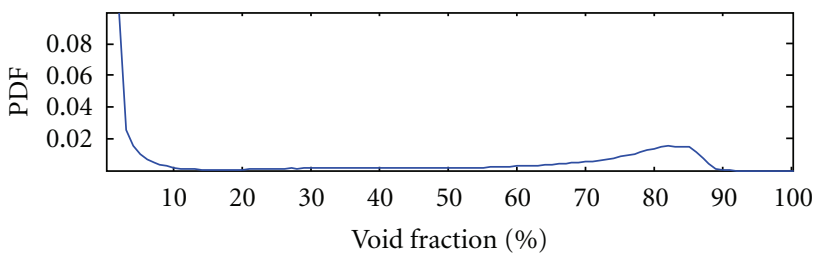

(c)

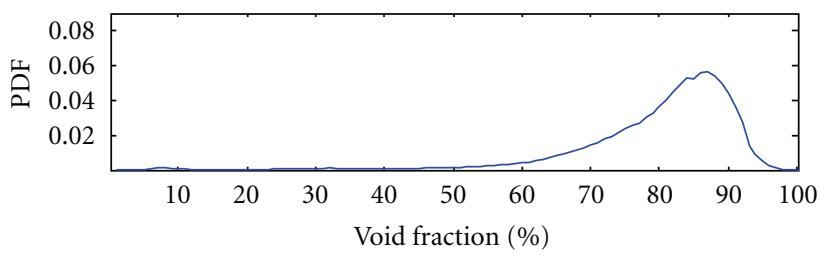

(e)

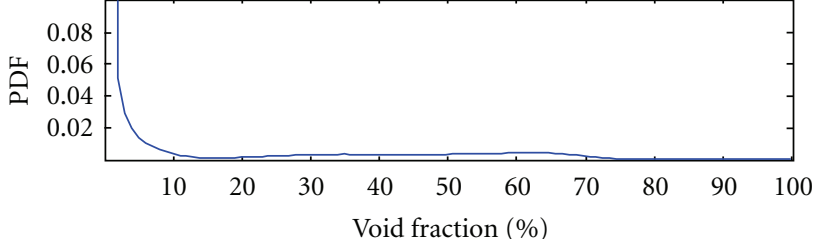

(b)

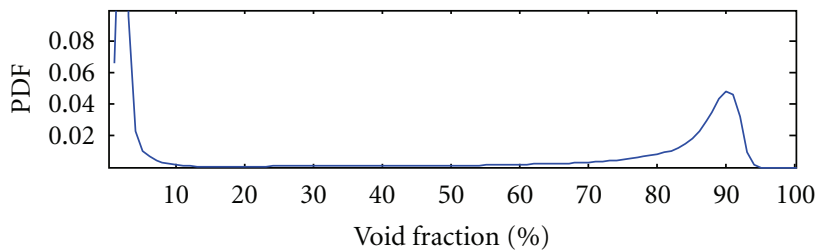

(d)

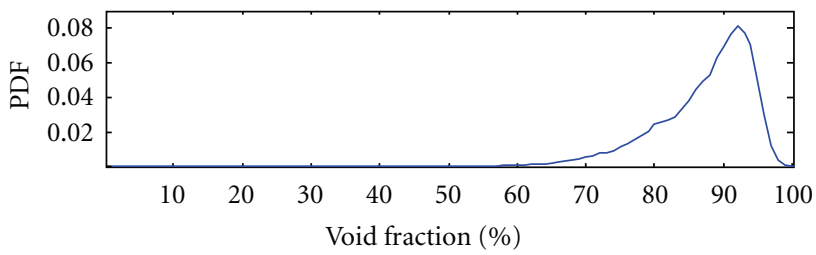

(f)

FIGURE 9: Distribution of the probability density function of the experimental void fraction time series for the various flow patterns as reported in Table 2: (a) bubble flow; (b) cap flow; (c) slug flow; (d) plug flow; (e) churn flow; (f) annular flow.

\section{Conclusions}

This study is concerned with the design, construction, and calibration of an impedance probe for the measure of void fraction in air-water two-phase flows. The main goal in the definition of the sensor has been devoted to achieve highspatial and -temporal resolution of the experimental void fraction time series, by means of appropriate geometrical design of the probe electrodes and electronic assessment of the data acquisition system. The adopted calibration procedure has been based on an estimation of void fraction through the implementation of a code for automatic extraction of bubble contours and the correction of image distortions.

For the entire spectrum of the possible flow patterns observed during the experimental campaign, the preliminary analysis in the time domain of the experimental time series detected by means of the void fraction probe, as well as the flow pattern classification based on the traditional PDF analysis, has pointed out the low influence of noise and the high-spatial and -temporal resolution of the experimental time series.

These are fundamental characteristics of the time series in view of the application of innovative tools of analysis oriented towards an accurate description of the complexity of nonlinear dynamical behaviour characterizing two-phase flow.

\section{References}

[1] T. Taitel, D. Bornea, and A. E. Dukler, "Modelling flow pattern transitions for steady upward gas-liquid flow in vertical tubes," AIChE Journal, vol. 26, no. 3, pp. 345-354, 1980.

[2] G. Costigan and P. B. Whalley, "Slug flow regime identification from dynamic void fraction measurements in vertical airwater flows," International Journal of Multiphase Flow, vol. 23, no. 2, pp. 263-282, 1997.

[3] Y. Mi, M. Ishii, and L. H. Tsoukalas, "Vertical two-phase flow identification using advanced instrumentation and neural networks," Nuclear Engineering and Design, vol. 184, no. 2-3, pp. 409-420, 1998.

[4] Y. W. Wang, B. S. Pei, and W. K. Lin, "Verification of using a single void fraction sensor to identify two-phase flow patterns," Nuclear Technology, vol. 95, no. 1, pp. 87-94, 1991.

[5] R. Van Hout, L. Shemer, and D. Barnea, "Spatial distribution of void fraction within a liquid slug and some other related slug parameters," International Journal of Multiphase Flow, vol. 18, no. 6, pp. 831-845, 1992.

[6] F. Devia and M. Fossa, "Design and optimisation of impedance probes for void fraction measurements," Flow Measurement and Instrumentation, vol. 14, no. 4-5, pp. 139-149, 2003.

[7] D. Lowe and K. S. Rezkallah, "A capacitance sensor for the characterization of microgravity two-phase liquid-gas flows," Measurement Science and Technology, vol. 10, no. 10, pp. 965$975,1999$.

[8] J. K. Keska and B. E. Williams, "Experimental comparison of flow pattern detection techniques for air-water mixture flow," 
Experimental Thermal and Fluid Science, vol. 19, no. 1, pp. 112, 1999.

[9] C. Vial, E. Camarasa, S. Poncin, G. Wild, N. Midoux, and J. Bouillard, "Study of hydrodynamic behaviour in bubble columns and external loop airlift reactors through analysis of pressure fluctuations," Chemical Engineering Science, vol. 55, no. 15, pp. 2957-2973, 2000.

[10] G. P. Lucas and I. C. Walton, "Flow rate measurement by kinematic wave detection in vertically upward, bubbly twophase flows," Flow Measurement and Instrumentation, vol. 8, no. 3-4, pp. 133-143, 1997.

[11] N. D. Jin, Z. Xin, J. Wang, Z. Y. Wang, X. H. Jia, and W. P. Chen, "Design and geometry optimization of a conductivity probe with a vertical multiple electrode array for measuring volume fraction and axial velocity of two-phase flow," Measurement Science and Technology, vol. 19, no. 4, 2008.

[12] T. J. Liu, "Bubble size and entrance length effects on void development in a vertical channel," International Journal of Multiphase Flow, vol. 19, no. 1, pp. 99-113, 1993.

[13] C. H. Song, M. K. Chung, and H. C. No, "Measurements of void fraction by an improved multi-channel conductance void meter," Nuclear Engineering and Design, vol. 184, no. 2-3, pp. 269-285, 1998.

[14] Y. Ma, N. Chung, B. Pei, and W. Lin, "Two simplified methods to determine void fractions for two-phase flow," Nuclear Technology, vol. 94, no. 1, pp. 124-133, 1991.

[15] M. J. Watson and G. F. Hewitt, "Pressure effects on the slug to churn transition," International Journal of Multiphase Flow, vol. 25, no. 6-7, pp. 1225-1241, 1999.

[16] P. Andreussi, A. Di Donfrancesco, and M. Messia, "An impedance method for the measurement of liquid hold-up in two-phase flow," International Journal of Multiphase Flow, vol. 14, no. 6, pp. 777-785, 1988.

[17] N. A. Tsochatzidis, T. D. Karapantsios, M. V. Kostoglou, and A. J. Karabelas, "A conductance probe for measuring liquid fraction in pipes and packed beds," International Journal of Multiphase Flow, vol. 18, no. 5, pp. 653-667, 1992.

[18] H. Yeung and A. Ibrahim, "Multiphase flows sensor response database," Flow Measurement and Instrumentation, vol. 14, no. 4-5, pp. 219-223, 2003.

[19] L. Cantelli, A. Fichera, I. D. Guglielmino, and A. Pagano, "Nonlinear dynamics of air-water mixtures in vertical pipes: experimental trends," International Journal of Bifurcation and Chaos, vol. 16, no. 9, pp. 2749-2760, 2006.

[20] J. Drahos, J. Tihon, C. Serio, and A. Lübert, "Deterministic chaos analysis of pressure fluctuations in a horizontal pipe at intermittent flow regime," The Chemical Engineering Journal, vol. 64, no. 1, pp. 149-156, 1996.

[21] N. D. Jin, X. B. Nie, Y. Y. Ren, and X. B. Liu, "Characterization of oil/water two-phase flow patterns based on nonlinear time series analysis," Flow Measurement and Instrumentation, vol. 14, no. 4-5, pp. 169-175, 2003.

[22] H. Letzel, J. Schouten, R. Krishna, and C. M. Van den Bleek, "Characterization of regimes and regime transitions in bubble columns by chaos analysis of pressure signals," Chemical Engineering Science, vol. 52, no. 24, pp. 4447-4459, 1997.

[23] S. F. Wang, R. Mosdorf, and M. Shoji, "Nonlinear analysis on fluctuation feature of two-phase flow through a T-junction," International Journal of Heat and Mass Transfer, vol. 46, no. 9, pp. 1519-1528, 2003.

[24] F. Franca, M. Acikgoz, R. T. Lahey Jr., and A. Clausse, "The use of fractal techniques for flow regime identification," International Journal of Multiphase Flow, vol. 17, no. 4, pp. 545-552, 1991. 

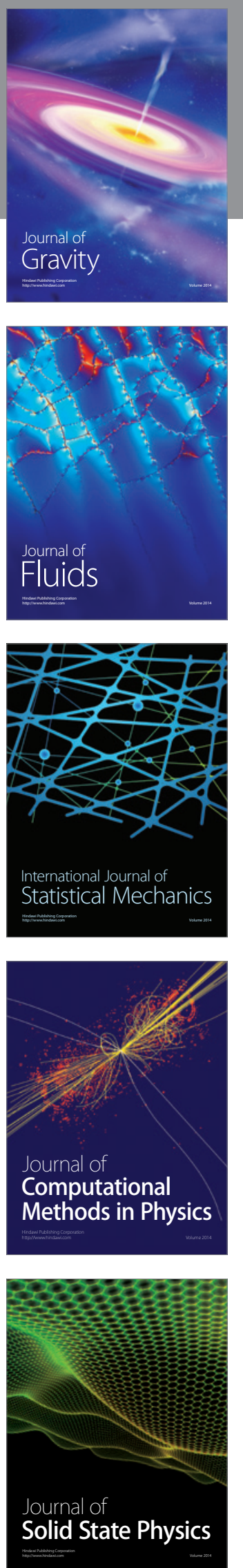

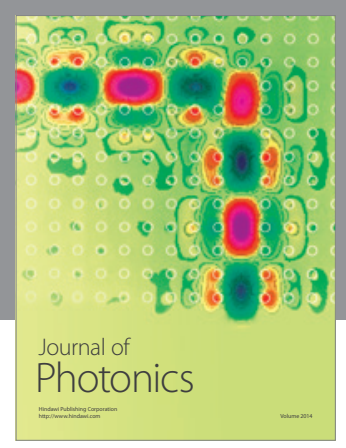

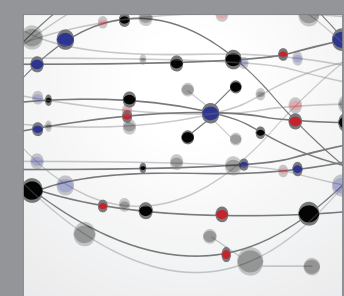

The Scientific World Journal
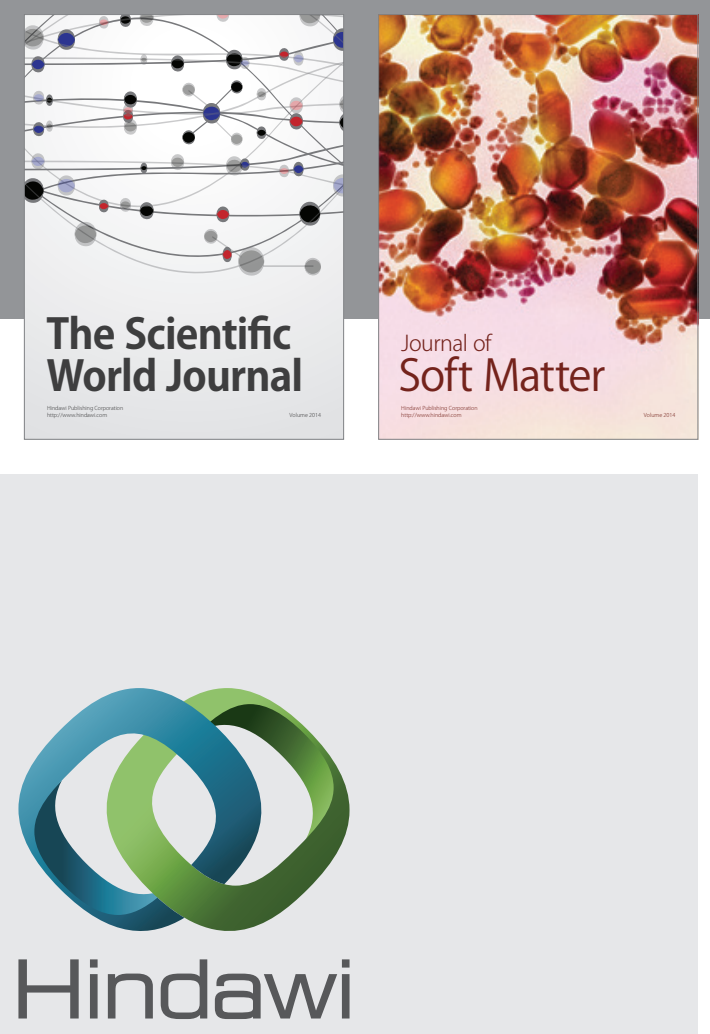

Submit your manuscripts at

http://www.hindawi.com
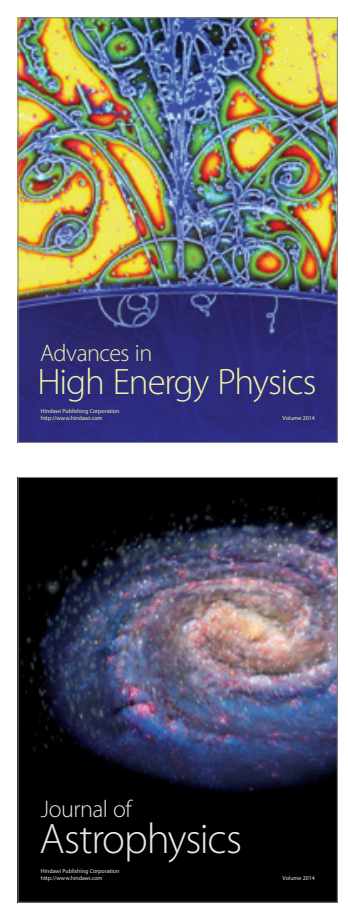
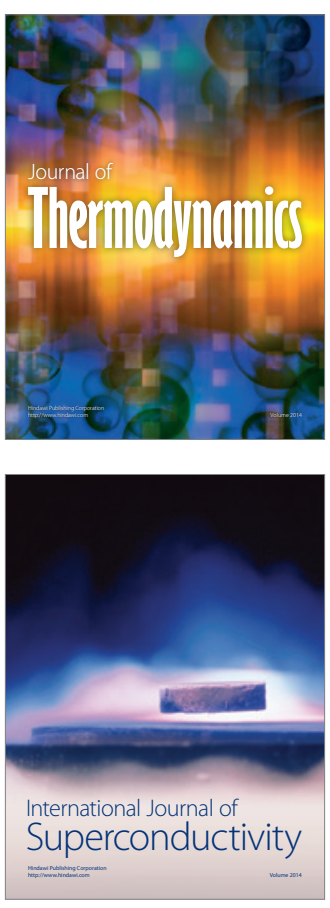
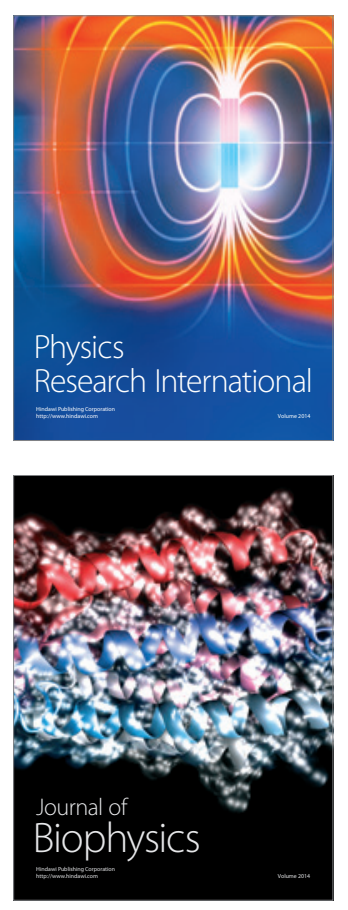
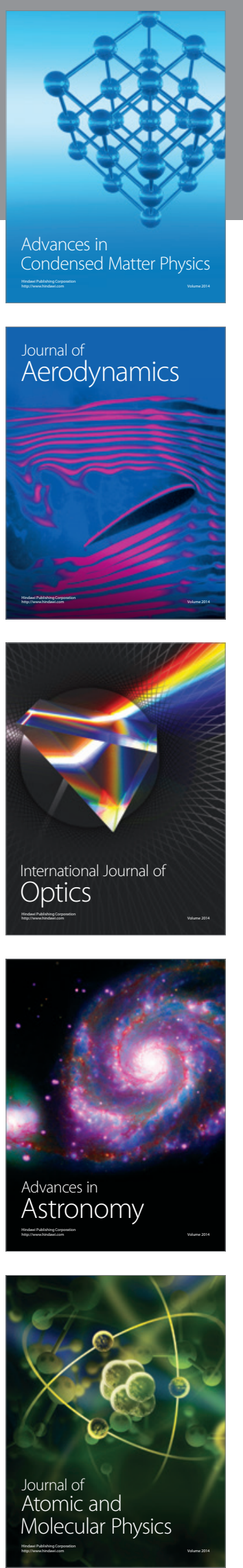